\title{
Trapping of Charge Carriers in Organic Molecular Materials: Phthalocyanine Thin Films Revisited
}

\author{
I. ZHIVKOV ${ }^{a}$, S. NEŠPUREK ${ }^{b}$ AND J. SWORAKOWSKI ${ }^{c, *}$ \\ ${ }^{a}$ Central Laboratory of Photoprocesses, Bulgarian Academy of Sciences \\ G. Bonchev Str., bl. 109, 1040 Sofia, Bulgaria \\ ${ }^{b}$ Institute of Macromolecular Chemistry, Academy of Sciences of the Czech Republic \\ Heyrovský Sq. 2, 16206 Prague 6, Czech Republic \\ and \\ Faculty of Chemistry, Technical University of Brno \\ Purkyňova 118, 61200 Brno, Czech Republic \\ ${ }^{c}$ Institute of Physical and Theoretical Chemistry, Technical University of Wroctaw \\ Wyb. Wyspiańskiego 27, 50-370 Wrocław, Poland
}

(Received November 14, 2001)

\begin{abstract}
The technique of temperature-modulated space-charge-limited currents was employed to study the energetic distribution of local states in thin films of metal-free phthalocyanine. Four well-defined local levels were detected within the energy range $0.5-0.3 \mathrm{eV}$, accompanied by an onset to a manifold of shallower states. The morphology of the films, depending on the deposition rate and changing from polycrystalline to nearly amorphous, affects the densities of local states but not their energies.
\end{abstract}

PACS numbers: 71.20.Rv, 72.20.Jv, 72.80.Le, 73.61.Ph

\section{Introduction}

A correct description of local states existing in all real dielectrics and wide-band-gap semiconductors (i.e. information about their densities, energetic and

*corresponding author; e-mail: sworakowski@kchf.ch.pwr.wroc.pl 
spatial distributions, etc.) is a basic requirement in characterizing transport and localization of charge carriers in low-conductivity materials (including molecular solids). A research into problems related to the injection, transport, and localization of charge carriers in molecular materials, extensively developed in the sixties and seventies and then somewhat neglected, has witnessed a revival due to emerging applications of these materials. The advent of organic light-emitting diodes (see the literature collected in recent reviews [1-3]), and recent papers by Schön, Kloc, and Batlogg (e.g., [4-9]) once again stimulated the interest of researchers.

Among the techniques commonly employed to study parameters of local states (e.g., thermally stimulated currents and thermally stimulated luminescence $[10,11]$, isothermal decay currents [12], transient and dc photocurrents [13, 14]), the technique of space-charge-limited (SCL) currents occupies a prominent position. Both steady-state and transient SCL currents have been studied for over half a century ([15, 16], see also $[17,18]$ and references therein). Correctly used, the method is a powerful tool allowing for the determination of energetic distributions of traps in insulators and wide-band-gap semiconductors. In particular, so-called "differential method" put forward in [19-21], and later developed into "temperature-modulated SCLC" (TMSCLC) [22-25] has been shown to provide information about details of distributions, inaccessible from other methods.

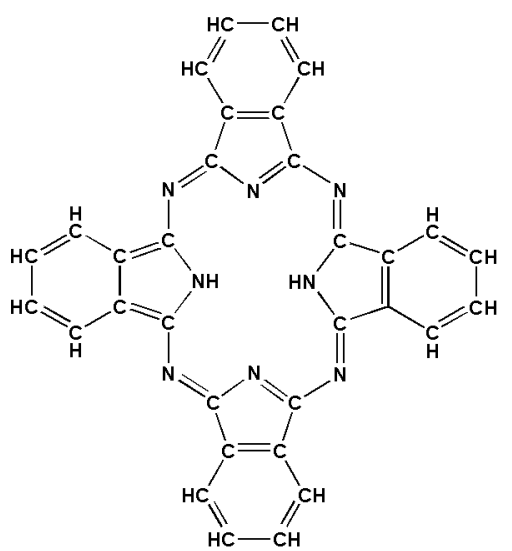

Fig. 1. The molecule of $\mathrm{H}_{2} \mathrm{Pc}$.

The aim of this paper is to present results of studies of local states in polycrystalline films of a model molecular semiconductor - metal-free phthalocyanine (Fig. 1) using the TMSCLC technique. Phthalocyanines are among prototypic organic semiconductors and their electrical properties have been studied since 1950s [26, 27]. Owing to their exceptional stability, phthalocyanines have been considered as promising materials suitable for applications in microelectronics (see, e.g., [28, 29] and literature therein). Recently, they have been used for 
the fabrication of FET transistors [30, 31], light-emitting diodes [32], gas sensors [33], photodetectors [34] and photoreceptors [35]. Most experiments have been carried out on copper phthalocyanine $(\mathrm{CuPc})$ and on metal-free phthalocyanine $\left(\mathrm{H}_{2} \mathrm{Pc}\right)$; in particular, SCLC experiments have been reported by several authors [24, 36-41] yielding results differing between various laboratories and often incompatible with those obtained from other techniques. The discrepancies may be partly attributed to the fact that, in spite of much effort, semiconductor properties of phthalocyanines are still difficult to control: conditions of fabrication of samples and their handling play an important role. Another factor is the incompatibility of the methods of interpretation of experimental results.

The organization of the paper is as follows: fundamentals of the TMSCLC method will be put forward in Sec. 2, focusing on underlying basic equations. Section 3 presents details of the experiments and data elaboration, followed by results and their interpretation grouped in Secs. 4 and 5. In particular, the attention will be paid to the effect of deposition conditions on the energetic structure of traps in vacuum-deposited thin films of $\mathrm{H}_{2} \mathrm{Pc}$.

\section{Steady-state space-charge-limited currents and the TMSCLC method}

\subsection{Basic equations of SCLC}

A necessary condition for the appearance of SCLC in an insulator is that the density of injected charge carriers, $n(x)$, should exceed that of thermally generated ones. Efficient injection of carriers needs that the sample be provided with an injecting ("ohmic") contact, and a neutral collecting one. Limiting ourselves to a plane-parallel geometry of the sample, we shall place the injecting contact at $x=0$, and the collecting one at $x=L, x$ standing for a linear coordinate and $L$ for the inter-electrode distance (here equal to the sample thickness). The existence of a perfect injecting contact requires that

$$
n(0)=\infty,
$$

and, consequently,

$$
F(0)=0 .
$$

In the above equations, $n$ and $F$ stand for the total concentration of injected carriers and the electric field. It is straightforward to demonstrate (see, e.g., [17]) that under these conditions both, the concentration of carriers and the field, will be functions of the linear coordinate $x$. Moreover, in real samples, the presence of traps localizing a fraction of injected carriers, is unavoidable. If the trapped carriers are in equilibrium with free ones, then relations between them may be obtained invoking a position-dependent quasi-Fermi level, $E_{\mathrm{F}}(x)$ (which is also a function of voltage - see below). In the case of wide-band-gap semiconductors and insulators, it is commonly assumed that the density of free carriers, $n_{\mathrm{f}}(x)$, can 
be described by the Boltzmann statistics, and the density of carriers localized by traps situated at an energy $E, n_{\mathrm{t}}(E, x)$, by the Fermi-Dirac statistics

$$
\begin{aligned}
& n_{\mathrm{f}}(x)=N_{\mathrm{b}} \exp \left(-\frac{E_{\mathrm{F}}(x)}{k T}\right), \\
& n_{\mathrm{t}}(E, x)=h(E)\left[1+\exp \left(\frac{E_{\mathrm{F}}(x)-E}{k T}\right)\right]^{-1} .
\end{aligned}
$$

In the above equations, $N_{\mathrm{b}}$ is the effective density of extended states, $T$ is the temperature, $k$ is the Boltzmann constant, and $h(E)$ is a function describing the energetic distribution of local states (DOS function)

$$
h(E)=\frac{\mathrm{d} H}{\mathrm{~d} E},
$$

$H$ being the total density of local states. The zero of the energy scale has been set at the band edge, its positive direction pointing towards the band. It should also be noted that, for the purpose of this paper, we shall assume the samples to be spatially homogeneous, i.e. $H \neq H(x)$ (for a discussion of the effect of spatial inhomogeneity see, e.g., [20, 21]).

SCL current-voltage characteristics can be derived upon solving the set of equations:

- the current equation

$j=e \mu n_{\mathrm{f}}(x) F(x)$,

- the Poisson equation

$$
\frac{\mathrm{d} F(x)}{\mathrm{d} x}=\frac{e n(x)}{\varepsilon}
$$

together with the boundary condition (2) and an equation relating $n_{\mathrm{f}}(x)$ and $n_{\mathrm{t}}(E, x)$. In the above equations, $j$ is the current density, $e$ is the unit charge, $\mu$ stands for the charge carrier mobility and $\varepsilon\left(=\varepsilon_{\mathrm{r}} \varepsilon_{0}\right)$ for the electric permittivity. Combining Eqs. (3) and (6), it is easy to demonstrate that the position of quasi-Fermi level is voltage-dependent. The equation describing the shift of the quasi-Fermi level at the collecting electrode, $E_{\mathrm{F}}(L)$, reads

$$
E_{\mathrm{F}}(L)=-k T \ln \frac{j}{U}+k T \ln \frac{e \mu N_{\mathrm{b}} \kappa_{1}}{L},
$$

where $\kappa_{1}$ is a parameter of the order of unity whose exact relation to experimental variables will be given later.

As was shown by Lampert and Mark [17], the voltage dependence of SCL current, $j(U)$, depends on the position of the quasi-Fermi level at the collecting electrode, $E_{\mathrm{F}}(L)$, with respect to energies of traps. If all traps at the collector are shallow (i.e., if $E_{\mathrm{F}}(L)$ is situated below the traps) or if they are completely filled 
(i.e., if $E_{\mathrm{F}}(L)$ is situated above the traps), then the current density can be written in the form

$$
j=\frac{9}{8} \mu \varepsilon \Theta \frac{U^{2}}{L^{3}},
$$

with

$$
\Theta=\frac{n_{\mathrm{f}}(L)}{n(L)},
$$

where the free-to-total carrier density ratio $\Theta \ll 1$ in the former case and $\Theta \approx 1$ in the latter case. If $E_{\mathrm{F}}(L)$ intersects the trap distribution, then the current-voltage characteristics is superquadratic, its shape depending on $h(E)$. In particular, for an exponential distribution of traps which will be used in Sec. 4 of this paper,

$$
h(E)=\frac{H}{k T_{\mathrm{c}}} \exp \left(\frac{E_{\mathrm{t}}-E}{k T_{\mathrm{c}}}\right) \quad\left(E>E_{\mathrm{t}}\right),
$$

the following equation is obtained [42]:

$$
j=N_{\mathrm{b}} \mu e^{1-l}\left(\frac{\varepsilon}{H} \frac{l}{l+1}\right)^{l}\left(\frac{2 l+1}{l+1}\right)^{l+1} \frac{U^{l+1}}{L^{2 l+1}} \exp \left(-\frac{E_{\mathrm{t}}}{k T}\right) .
$$

In the above equations, $E_{\mathrm{t}}$ is a cut-off energy (often assumed equal to 0 ), $T_{\mathrm{c}}$ is a parameter (so-called characteristic temperature), and $l=T_{\mathrm{c}} / T>1$. Because of simplicity of the analytical solution, the exponential distribution of traps in energy is often used to interpret experimental results obtained on both, amorphous inorganic semiconductors [43] and molecular organic materials [44]. In many cases, however, it would be more appropriate to invoke a Gaussian distribution of traps [45], having a better physical justification

$$
h(E)=\frac{H}{(2 \pi)^{1 / 2} \sigma} \exp \left[-\frac{\left(E_{\mathrm{t}}-E\right)^{2}}{2 \sigma^{2}}\right],
$$

where $\sigma$ is the distribution variance, related to its width. For a more extensive discussion see, e.g., $[45,46]$.

It should be reminded that the given above equations have been derived for plane-parallel arrangement of electrodes. As the samples investigated in this work were measured in the surface arrangement (cf. Fig. 2), a question should be answered whether the equations can be applied to interpret our experiments. This problem arises in the interpretation of results obtained for all devices operating in surface geometry, e.g., FET structures. In this case it is necessary to take into consideration not only component of the electric field in the direction of the current flow (parallel to the substrate, $x$ direction), but also the transverse component (perpendicular to the substrate, $y$ direction). The "active thickness" of the sample (i.e., region of the sample directly beneath the electrodes approximately equal to the penetration depth of the field along $y$ ) is difficult to assess, in particular for anisotropic samples. 

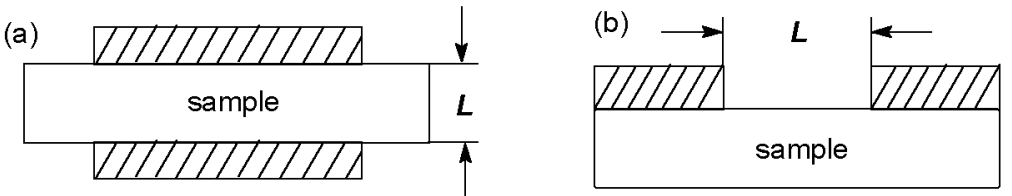

Fig. 2. Electrode configurations for a thin layer: (a) a sandwich-type structure, (b) a gap-type structure. The electrodes have been hatched, $L$ stands for the inter-electrode distance.

The problem of SCL currents in the surface geometry was tackled only for a trap-free case or for the currents controlled by shallow traps. According to [47], Eq. (9) should in this case be modified to

$$
j=\frac{2}{\pi} \mu \varepsilon \Theta \frac{U^{2}}{L^{2}}
$$

$j$ being a "surface current density" $[\mathrm{A} / \mathrm{m}]$, and $L$ standing for the electrode spacing. Note that the equation predicts a dependence on $L$ different from that expected from Eq. (9). Such a dependence was indeed found in $\beta$-metal-free phthalocyanine crystals [48].

2. . Temperature-modulated space-charge-limited current method

As comes from the above discussion, it is in principle possible to deduce the shape of a DOS function from the shape of an SCL current-voltage characteristic, $j(U)$. This method, however, requires that $h(E)$ be assumed a priori and generally yields results of a doubtful reliability. As early as in the seventies and early eighties, methods were put forward allowing for a direct determination of DOS functions from measurements of SCLC without a priori assumptions about the shapes of the distributions $[19,49-51]$. The methods were based on differentiation of SCL current-voltage characteristics and assumed a zero-temperature statistics, i.e. the quasi-Fermi level was taken as a sharp demarcation level between filled and empty traps. The latter assumption results, however, in problems with setting the energy scale. Limitations of the differential method were overcome in the method referred to as temperature-modulated space-charge-limited-current spectroscopy [22-25]. The method consists in simultaneous measurements of steady-state SCL current-voltage and activation energy-voltage dependences. Basic features of the method will be presented below; for a more detailed description, the reader is referred to the original literature.

For any $E_{\mathrm{F}}$, the total concentration of carriers (in most cases practically equal to the concentration of trapped carriers) is given by the equation

$$
n=\int_{E} h(E) f\left(E-E_{\mathrm{F}}\right) \mathrm{d} E
$$

where $f\left(E-E_{\mathrm{F}}\right)$ is the Fermi-Dirac function (cf. Eq. (4)). In the following discussion we shall implicitly assume $x=L$; hence $n \equiv n(L), E_{\mathrm{F}} \equiv E_{\mathrm{F}}(L)$, etc. Upon 
changing the voltage, $E_{\mathrm{F}}$ moves from its "thermodynamic" value towards the edge of extended states (cf. Eq. (8)) scanning local states and changing their occupancy

$$
\frac{\mathrm{d} n}{\mathrm{~d} E_{\mathrm{F}}}=\frac{\mathrm{d}}{\mathrm{d} E_{\mathrm{F}}}\left(\int_{E} n(E) \mathrm{d} E\right)=\int_{E} h(E) \frac{\mathrm{d} f\left(E-E_{\mathrm{F}}\right)}{\mathrm{d}\left(E-E_{\mathrm{F}}\right)} \mathrm{d} E .
$$

Within the zero-temperature approximation [19], the energy scale would be set by Eq. (8), with $\kappa_{1}=m /(2 m-1), m$ standing for the slope of the current-voltage characteristic in the $\log -\log$ coordinates $(m=\mathrm{d}(\ln j) / \mathrm{d}(\ln U))$. This approach, however, may result in substantial errors [24], hence it is useful to introduce the notion of a dominant energy level, $E_{\mathrm{d}}[52]$, setting the energy scale. Mathematically, $E_{\mathrm{d}}$ represents the first momentum of the energy distribution of trapped carriers [53]

$$
E_{\mathrm{d}}=\frac{\int_{E} h(E) f\left(E_{\mathrm{F}}-E\right)\left[1-f\left(E_{\mathrm{F}}-E\right)\right] E \mathrm{~d} E}{\int_{E} h(E) f\left(E_{\mathrm{f}}-E\right)\left[1-f\left(E_{\mathrm{F}}-E\right)\right] \mathrm{d} E} .
$$

At any voltage, the position of the dominant level is related to the experimentally determined activation energy of the current, as will be shown later.

\section{Experimental procedures}

\subsection{The samples}

$\mathrm{H}_{2} \mathrm{Pc}$ powder was vacuum purified by a triple sublimation in a temperature gradient. Gold coplanar electrodes separated by $13 \mu \mathrm{m}$ gap were vacuum deposited on Corning 7059 glass substrates. In a series of experiments, films of thicknesses ranging between 30 and $1300 \mathrm{~nm}$ were vacuum evaporated at a constant rate of $(14.0 \pm 2.5) \mathrm{nm} \mathrm{min}{ }^{-1}$, whereas another set of samples of a nearly constant thickness $(1000 \pm 100) \mathrm{nm}$ was prepared employing various evaporation rates from 20 to $900 \mathrm{~nm} \mathrm{~min}^{-1}$. The films obtained consisted of crystallites of $\alpha$-phase of $\mathrm{H}_{2} \mathrm{Pc}$, as was checked by UV-VIS spectroscopy. Scanning electron microscope (SEM) images reveal that the samples consist of well-oriented crystallites (see Fig. 3) whose orientation indicates that columns of $\mathrm{H}_{2} \mathrm{Pc}$ molecules are nearly perpendicular to the substrates. Increasing the deposition rate results in a decrease in the size of the surface grains and, consequently, in change of the film structure from polycrystalline to quasi-amorphous. Further SEM experiments performed on samples deposited at a constant rate of $(14.0 \pm 2.5) \mathrm{nm} \mathrm{min}^{-1}$ show that upon increase in the thickness from 30 to $1300 \mathrm{~nm}$ the upper surfaces of the samples become more coarse-grained.

\subsection{Measurements}

After mounting a sample for TMSCLC measurements, the measuring chamber was pumped down to the pressure of $7 \mathrm{~Pa}$, then filled with nitrogen to the atmospheric pressure. This procedure was repeated three times. The sample was 

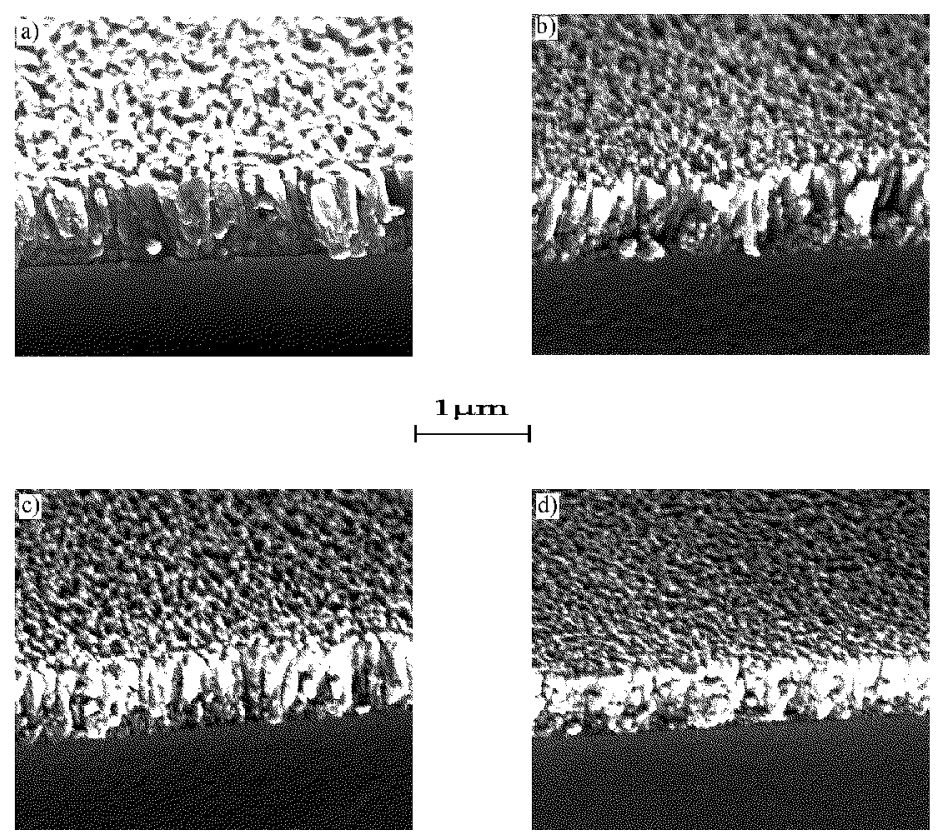

Fig. 3. Cross-sectional SEM images of thin films of $\mathrm{H}_{2} \mathrm{Pc}$ deposited at various rates: (a) $15 \mathrm{~nm} / \min$, (b) $50 \mathrm{~nm} / \min$, (c) $90 \mathrm{~nm} / \mathrm{min}$, (d) $490 \mathrm{~nm} / \mathrm{min}$.

then heated in the dark at $413 \mathrm{~K}$ to remove adsorbed oxygen. TMSCLC characteristics were measured in the dark, under nitrogen at atmospheric pressure.

A reliable determination of DOS and the energy scale requires that $j(U)$ and $E_{\mathrm{a}}(U)$ dependencies be measured simultaneously. Therefore, the following experimental procedure has been employed: at a constant temperature $T_{1}$ (typically $298 \mathrm{~K}$ ), a certain voltage, $U_{1}$, is applied to the sample and, after a time necessary to attain the steady state (i.e. to reach an equilibrium between free and trapped carriers), the temperature is slowly changed to $T_{2}\left(\Delta T=T_{2}-T_{1}\right.$ amounts typically to ca. $3-5 \mathrm{~K}$ ). During the temperature run the current is measured and its activation energy $E_{\mathrm{a} 1}$ is determined from the $\ln j$ vs. $1 / T$ dependence. Then using $E_{\mathrm{a} 1}$ and the average temperature $\left(T_{2}+T_{1}\right) / 2$, the average current $j_{1}$ is calculated. In such a way the first point of the TMSCLC experiment $\left(U_{1}, j_{1}, E_{\mathrm{a} 1}\right)$ is obtained. One then returns to the temperature $T_{1}$, the voltage on the sample is changed to $U_{2}$ and the whole procedure is repeated.

\subsection{Elaboration of experimental data}

The essence of the TMSCLC method rests in calculating the DOS function by a deconvolution of the integral in Eq. (16). The algorithm of the deconvolution, put forward in [24], will be shortly presented below.

The total concentration of carriers (cf. Eq. (15)) for any $E_{\mathrm{F}}$ can be related to experimental TMSCLC data via the equation 


$$
n=\frac{\varepsilon U}{e L^{2}}(2-\gamma)(1-\gamma)(1+B)
$$

where $\gamma$ is the inverse of the slope of the current-voltage characteristic, $\gamma=$ $1 / m=\mathrm{d}(\ln U) / \mathrm{d}(\ln j) \leq 1 / 2$, and $B$ is a parameter containing higher order corrections

$$
B=\frac{\mathrm{d} \gamma / \mathrm{d}(\ln j)}{(2-\gamma)(1-\gamma)}
$$

The DOS function is related to the concentration of carriers by the equation

$$
h(E)=\frac{\mathrm{d} n}{\mathrm{~d} E_{\mathrm{F}}}-\frac{\mathrm{d}^{3} n}{\mathrm{~d} E_{\mathrm{F}}^{3}} M_{2},
$$

where the increment of the carrier concentration, $\mathrm{d} n / \mathrm{d} E_{\mathbf{F}}$, is connected with experimentally determined quantities via Eqs. (18) and (8), and

$$
M_{2}=\int_{-\infty}^{+\infty}\left(E_{\mathrm{F}}-E\right)^{2} f\left(E-E_{\mathrm{F}}\right)\left[1-f\left(E-E_{\mathrm{F}}\right)\right] \mathrm{d} E \approx 3.2898(k T)^{2}
$$

is a temperature-dependent constant. Note that at low temperatures $(T \rightarrow 0 \mathrm{~K})$ the density of states is just equal to $\mathrm{d} n / \mathrm{d} E_{\mathrm{F}}$, as was postulated in earlier papers [19-21]. As the latter increment can vary by several orders of magnitude, it is more convenient to deconvolute the logarithmic form of Eq. (15). An equivalent of Eq. (20) can then be written as

$$
h(E)=\frac{\mathrm{d} n}{\mathrm{~d} E_{\mathrm{F}}}\left[1-\left(\rho^{2}+\rho^{\prime}\right) M_{2}\right]
$$

where $\rho=\mathrm{d}\left[\ln \left(\mathrm{d} n / \mathrm{d} E_{\mathrm{F}}\right) / \mathrm{d} E_{\mathrm{F}}\right]$, and $\rho^{\prime}=\mathrm{d} \rho / \mathrm{d} E_{\mathrm{F}}$.

The position of the dominant level determining the energy scale, is related to the experimentally determined activation energy of the current by the equation

$$
E_{\mathrm{d}}=E_{\mathrm{a}}+\frac{(3 \gamma-4) \gamma}{(2-\gamma)(1-\gamma)} \frac{\mathrm{d} E_{\mathrm{a}}}{\mathrm{d}(\ln j)}+\frac{1}{1+B} \frac{\mathrm{d} B}{\mathrm{~d}(1 / k T)}
$$

where the parameters $\gamma$ and $B$ have been defined above. The last term in Eq. (23) contains higher-order corrections and can be neglected without any substantial loss of accuracy.

The above equations can be employed to interpret experimental data: one can use experimentally determined values of the activation energy of the current, its derivative $\mathrm{d} E_{\mathrm{a}} / \mathrm{d}(\ln j)$ and the inverse of the slope of the current-voltage characteristic, $\gamma=\mathrm{d}(\ln U) / \mathrm{d}(\ln j)$. At each voltage, the dominant energy can be calculated from Eq. (23) and DOS from Eq. (22). Before taking the derivatives, the characteristics must be smoothed to minimize the experimental scatter. The smoothing was done by cubic splines as were calculations of the derivatives. 


\section{Results}

Figure 4a shows results of a series of TMSCLC experiments carried out on samples of various thicknesses ranging between 50 and $1300 \mathrm{~nm}$, deposited at the same rate of $(14 \pm 2.5) \mathrm{nm} \mathrm{min}^{-1}$. At a first sight, the current-voltage characteristics shown in Fig. 4 a seem to fulfil a $j \propto U^{3}$ dependence, apparently following $\mathrm{Eq}$. (12) with $T_{\mathrm{c}} \approx 600 \mathrm{~K}$. The DOS functions calculated from the experimental results shown in Fig. 4a are shown in Fig. 4b. Use of the TMSCLC method reveals the presence of 4 relatively well defined peaks at $0.34,0.41,0.45$, and $0.49 \mathrm{eV}$, and an onset to a shallower distribution.
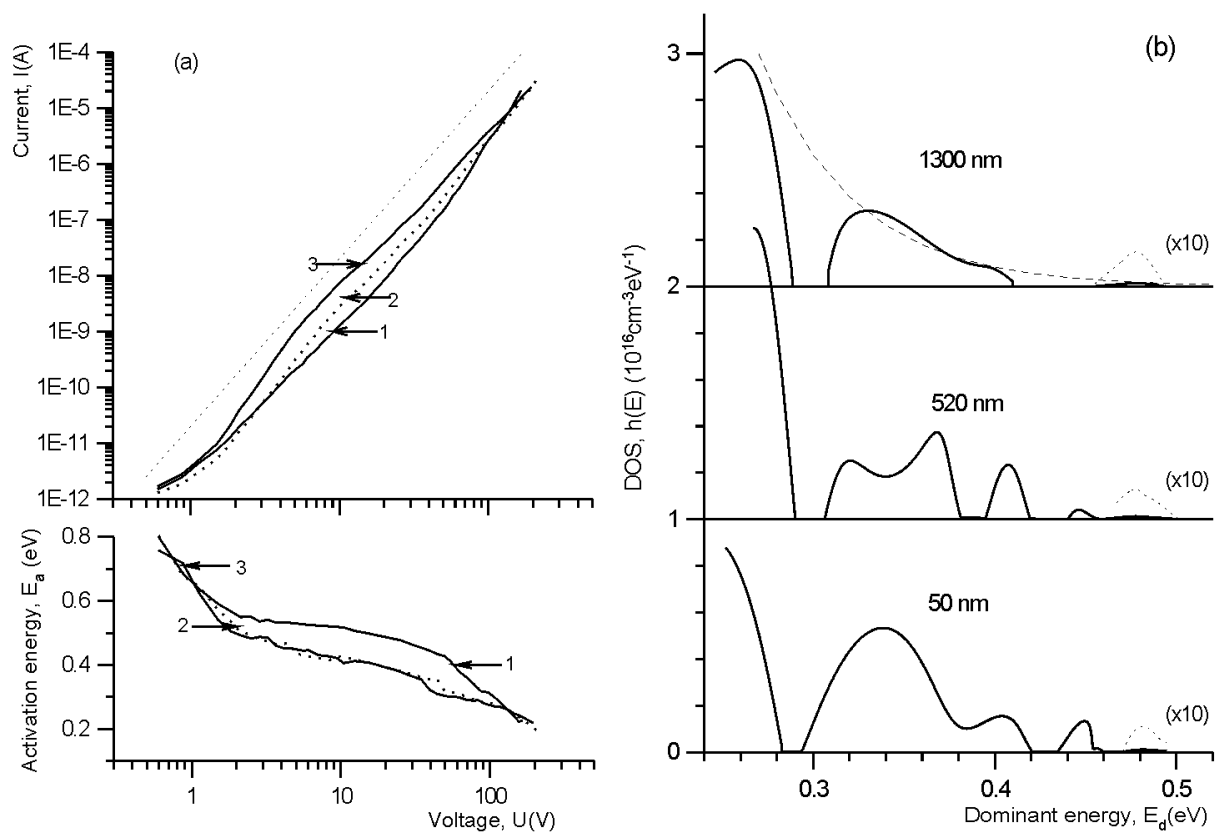

Fig. 4. (a) Current-voltage and activation energy-voltage dependencies measured on samples deposited at a constant rate $(14.0 \pm 2.5) \mathrm{nm} / \mathrm{min}$. The thicknesses amount to 50,520 , and $1300 \mathrm{~nm}$ for the curves 1 to 3 , respectively. The dashed line over the characteristics marks a $j \propto U^{3}$ dependence. (b) DOS functions calculated from the experimental data. The functions have been vertically displaced for the sake of clarity. The dashed curve in the uppermost part of the figure is the exponential distribution calculated from Eq. (11) with $T_{\mathrm{c}}=607 \mathrm{~K}$.

The effect of the deposition rate is shown in Fig. 5. The samples investigated were of approximately the same thickness $(1000 \pm 100) \mathrm{nm}$ deposited at several different rates. The analysis of experimental results again reveals the presence of 

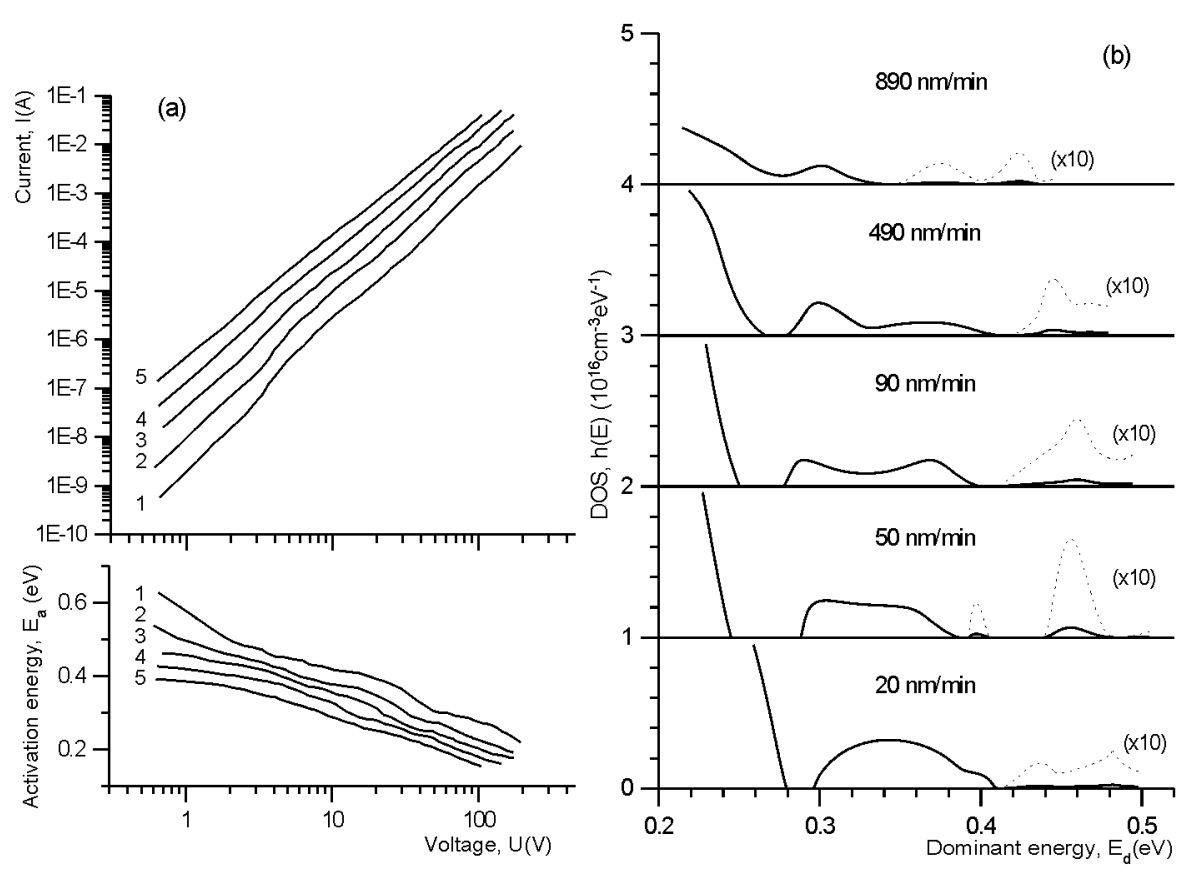

Fig. 5. (a) Current-voltage and activation energy-voltage dependencies measured on samples ca. $1000 \mathrm{~nm}$ thick, deposited at various rates. The rates amount to 20, 50, 90,490 , and $890 \mathrm{~nm} / \mathrm{min}$ for curves 1 to 5 , respectively. (b) DOS functions calculated from the experimental data. The functions have been vertically displaced for the sake of clarity.

a few peaks between 0.3 and $0.5 \mathrm{eV}$ and an onset to a shallower distribution, the differences in the heights of the peaks being now more pronounced than in the previous series of experiments.

\section{Discussion and conclusions}

The results reported in this paper have been obtained employing the TMSCLC method. We believe that its superiority over the conventional SCLC has been convincingly demonstrated. One should, however, comment on the problem of sample geometry, mentioned in Sec. 3. The results shown in Fig. 4a clearly demonstrate that the dependence of both, the current and the activation energy, on the thicknesses of evaporated films is rather weak. One may thus infer that the effect of the transverse electric field is not important and the carriers are transported in a thin layer close to the substrate; consequently, the inter-electrode distance can indeed be taken for the parameter $L$ and all calculations can be performed employing the equations given in Sec. 3. This simplification results in a 
small relative shift of the DOS curves between the samples of various thicknesses which should be corrected after all calculations have been performed. It is interesting to note that, although the uncertainty in setting the energy scale may be estimated to be $c a .0 .05 \mathrm{eV}$, the sequence of major features of the DOS functions measured on various samples appears in a quite reproducible way.

Our experiments revealed the existence of 4 closely lying nearly discrete trapping levels located between ca. 0.3 and $0.5 \mathrm{eV}$, and an onset to a shallower distribution of local states. One may suppose that the latter states are just tail states merging into the extended (band) states. The existence of some states located in the energy range covered by our experiments has been found previously in independent experiments. The traps $(0.37 \pm 0.03) \mathrm{eV}$ and $0.3 \mathrm{eV}$ deep were detected in sandwich samples [54] and in single crystals [55] of $\mathrm{H}_{2} \mathrm{Pc}, \mathrm{ZnPc}, \mathrm{CoPc}, \mathrm{NiPc}$, and $\mathrm{CuPc}$ by the method of thermally stimulated currents. A set of traps located at $0.25,0.36,0.50$, and $0.58 \mathrm{eV}$ was detected in sandwich structure $\mathrm{CdS} / \mathrm{H}_{2} \mathrm{Pc} / \mathrm{Au}$, using TMSCLC [24]. The conventional SCLC technique applied to single crystals of $\mathrm{H}_{2} \mathrm{Pc}$ yielded traps $0.38 \mathrm{eV}$ [40] and $0.32 \mathrm{eV}$ [41]. From the activation energy of the photoconductivity, a trap level located at $0.21 \mathrm{eV}$ was detected in $\beta-\mathrm{H}_{2} \mathrm{Pc}$ [56].

Interesting conclusions can be drawn from a comparison of the morphology of the samples and the shape of the DOS function. The samples deposited at the lowest rates consist of well-oriented crystallites, typically a fraction of micrometer thick, whereas those deposited at high evaporation rates are nearly amorphous. This difference of morphologies reflects itself in the shape of the DOS functions calculated from our experiment: as appears from a comparison of Figs. 3 and 5b, the decrease in the size of crystallites apparently coincides with a decrease in the concentration of local states. Positions of the maxima seem to shift towards lower energies, though their sequence remains almost unchanged. This feature can be rationalized assuming that either the source of traps should be sought inside the crystallites or the SCLC characteristics measured in our experiments are controlled by states due to some type of interactions of the crystallites with the substrate. The energy shift may also result from the Meyer-Neldel effect [57]. A more detailed discussion of the origin of local states in molecular semiconductors and insulators is beyond the scope of this paper; the reader is referred to, e.g., [14, 58].

Finally, it should be pointed out that the expressions discussed in Sec. 2 have been derived for the charge carrier transport in the band. In disordered molecular solids, where the carrier transport occurs mainly via hopping in tail states, a correction in the expression for dominant energy is necessary. This problem, as well as implications of the Meyer-Neldel effect, will be discussed in detail elsewhere.

\section{Acknowledgments}

The work was supported by the Grant Agency of the Academy of Sciences of the Czech Republic (grants No. 1050901 and AV0Z4050913), by Grant Agency 
of the Czech Republic (grant No. 202/01/0518) and by the Technical University of Wrocław. The experiments were carried out while one of the authors (I.Z.) was with the Institute of Macromolecular Chemistry of CAS supported by the Grant Agency of the Czech Republic.

\section{References}

[1] J. Kalinowski, J. Phys. D, Appl. Phys. 32, R179 (1999).

[2] P.W.M. Blom, M.J.C.M. Vissenberg, Mater. Sci. Eng. R 27, 53 (2000).

[3] U. Mitschke, P. Bäuerle, J. Mat. Chem. 10, 1471 (2000).

[4] J.H. Schön, Ch. Kloc, R.A. Laudise, B. Batlogg, Phys. Rev. B 58, 12952 (1998).

[5] J.H. Schön, Ch. Kloc, E. Bucher, B. Batlogg, Nature 403, 408 (2000).

[6] J.H. Schön, S. Berg, Ch. Kloc, B. Batlogg, Science 287, 1022 (2000).

[7] J.H. Schön, Ch. Kloc, B. Batlogg, Science 288, 2338 (2000).

[8] J.H. Schön, Ch. Kloc, A. Dodabalapur, B. Batlogg, Science 289, 599 (2000).

[9] J.H. Schön, Ch. Kloc, B. Batlogg, Appl. Phys. Lett. 77, 3776 (2000).

[10] Thermally Stimulated Relaxation in Solids, Ed. P. Bräunlich, Springer, Berlin 1979.

[11] R. Chen, Y. Kirsh, Analysis of Thermally Stimulated Processes, Pergamon, Oxford 1981.

[12] J.G. Simmons, M.C. Tam, Phys. Rev. B 7, 3706 (1973).

[13] R.H. Bube, Photoconductivity in Solids, Wiley, New York 1960.

[14] M. Pope, C.E. Swenberg, Electronic Processes in Organic Crystals and Polymers, 2nd ed., Oxford Univ. Press, Oxford 1999.

[15] A. Rose, Phys. Rev. 97, 1538 (1955).

[16] M.A. Lampert, Phys. Rev. 103, 1648 (1956).

[17] M.A. Lampert, P. Mark, Current Injection in Solids, Academic Press, New York 1970 .

[18] K.C. Kao, W. Hwang, Electrical Transport in Solids, Pergamon, Oxford 1981.

[19] S. Nešpurek, J. Sworakowski, J. Appl. Phys. 51, 2098 (1980).

[20] J. Sworakowski, S. Nešpurek, IEEE Trans. Electr. Ins. 24, 223 (1989).

[21] J. Sworakowski, S. Nešpurek, J. Appl. Phys. 65, 1559 (1989).

[22] F. Schauer, V. Šmíd, O. Zmeškal, L. Štourač, Phys. Status Solidi A 73, K199 (1982).

[23] O. Zmeškal, F. Schauer, S. Nešpurek, J. Phys. C, Solid State Phys. 18, 1873 (1985).

[24] F. Schauer, S. Nešpurek, O. Zmeškal, J. Phys. C, Solid State Phys. 19, 7231 (1986).

[25] F. Schauer, S. Nešpurek, R. Novotny, J. Appl. Phys. 81, 1244 (1997).

[26] D.D. Eley, G.D. Parfitt, Trans. Faraday Soc. 51, 1529 (1956).

[27] P.E. Fielding, F. Gutman, J. Chem. Phys. 26, 411 (1957). 
[28] Phthalocyanines, Eds. C.C. Leznoff, A.B.P. Lever, Vol. 1-4, VCH, Weinheim $1989-1996$.

[29] G. Guillaud, J. Simon, J.P. Germain, Coordination Chem. Revs. 178-180, 1433 (1998).

[30] Z. Bao, A.J. Lovinger, A. Dodabalapur, Appl. Phys. Lett. 69, 3066 (1996).

[31] I. Zhivkov, S. Nešpurek, F. Schauer, Adv. Mater. Opt. Electron. 9, 175 (1999).

[32] A. Fujii, M. Yoshida, Y. Ohmori, K. Yoshino, Jpn. J. Appl. Phys. 35, L37 (1996).

[33] P. Roisin, J.D. Weight, R.J.M. Nolte, O.E. Sieicken, S.C. Thorpe, J. Mater. Sci. 2, 131 (1992).

[34] H.J. Wagner, R.O. Loutfy, J. Vac. Sci. Technol. 20, 300 (1982).

[35] P. Haisch, G. Winter, M. Hanock, Adv. Mater. 9, 316 (1997).

[36] G.M. Delacôte, J.P. Fillard, F.J. Marco, Solid State Commun. 2, 373 (1964).

[37] A. Sussman, J. Appl. Phys. 38, 2738 (1967).

[38] C. Hamann, Phys. Status Solidi 20, 481 (1967).

[39] C. Hamann, Phys. Status Solidi 26, 311 (1968).

[40] D.F. Barbe, C.R. Westgate, Solid State Comun. 7, 563 (1969).

[41] G.A. Cox, P.C. Knight, J. Phys. Chem. Solids 34, 1655 (1973).

[42] P. Mark, W. Helfrich, J. Appl. Phys. 33, 205 (1962).

[43] R.A. Street, Hydrogenated Amorphous Silicon, Cambridge Univ. Press, Cambridge 1991.

[44] W. Helfrich, in: Physics and Chemistry of the Organic Solid State, Eds. D. Fox, M.M. Labes, A. Weissberger, Vol. 3, Interscience, New York 1967.

[45] J.S. Bonham, Austral. J. Chem. 26, 927 (1973).

[46] S. Nešpurek, E.A. Silinsh, Phys. Status Solidi A 34, 747 (1976).

[47] J.A. Geurst, Phys. Status Solidi 15, 107 (1966).

[48] D.F. Barbe, C.R. Westgate, J. Chem. Phys. 52, 4046 (1970).

[49] J.S. Pfister, Phys. Status Solidi A 24, K15 (1974).

[50] C. Manfredotti, C. De Blasi, S. Galassini, G. Micocci, L. Ruggiero, A. Tepore, Phys. Status Solidi A 36, 569 (1976).

[51] F. Stöckmann, Phys. Status Solidi A 64, 475 (1981).

[52] G.G. Roberts, F.W. Schmidlin, Phys. Rev. 180, 785 (1969).

[53] M. Kikuchi, J. Appl. Phys. 64, 4997 (1988).

[54] K. Yoshino, K. Kento, K. Tatsuno, Y. Inushi, Technol. Rep. Osaka Univ. 22, 585 (1972).

[55] C.R. Westgate, G. Warfield, J. Chem. Phys. 46, 537 (1967).

[56] G.A. Cox, P.C. Knight, J. Phys. C, Solid State Phys. 7, 146 (1974).

[57] W. Meyer, H. Nelder, Z. Tech. Phys. 12, 588 (1937).

[58] E.A. Silinsh, Organic Molecular Crystals. Their Electronic States, Springer, Berlin 1980 . 